Cite this: Phys. Chem. Chem. Phys., 2013,

\title{
Impact of long-range van der Waals forces on chiral recognition in a Cinchona alkaloid chiral selector system $\dagger$
}

15, 6113

\author{
Petr Milko, $\ddagger^{\star a}$ Jana Roithová, ${ }^{a}$ Kevin A. Schug ${ }^{b}$ and Karel Lemr ${ }^{c}$
}

Singly-charged complexes of $(8 S, 9 R)$-tert-butylcarbamoylquinine (tBuCQN), N-3,5-dinitrobenzoyl-( $S, R)$-leucine (DNB-S/R-leucine), and alkali metal counter ions $\left(\mathrm{Li}^{+}, \mathrm{Na}^{+}, \mathrm{K}^{+}\right)$were investigated by density-functional theory. It is shown that the cations prefer formation of an ionic pair with the carboxylate group of DNB-Leu over the formation of a cation- $\pi$ interaction. The [tBuCQN.DNB-S/R-Leu.Na] $]^{+}$complex is bound by a Coulombic attraction, a hydrogen bond, a $\pi-\pi$ interaction and van der Waals forces. The $t B u C Q N$ chiral selector preferentially complexes with the DNB-S-Leu enantiomer, because the favourable stereochemistry allows the stabilization of the complex by at least one binding mode more compared to the complex containing the DNB-R-Leu molecule. Weakening of the binding modes is observed using the lithium counter ion compared to the sodium one. The weakening is more pronounced in [tBuCQN.DNB-R-Leu.Li] $]^{+}$than in [tBuCQN.DNB-S-Leu.Li] ${ }^{+}$. The exact opposite effect is observed using the potassium counter ion. Hence, the lithium counter ion enhances the enantioselectivity of $t B u C Q N$ while the potassium counter ion reduces the enantioselectivity of $t B u C Q N$. www.rsc.org/pccp

Received 10th December 2012, Accepted 25th February 2013

DOI: $10.1039 / \mathrm{c} 3$ cp44444a

\section{Introduction}

Today, chiral compounds are used in large scales and in many fields of chemistry. ${ }^{1,2}$ Along with this, the identification and separation of enantiomers are important processes. High-performance liquid chromatography (HPLC) can be considered as one of the most popular techniques for chiral separations and it is considered as a reliable tool for direct chiral separations if appropriate chiral stationary phases (CSPs) are available. ${ }^{3}$ Synthesis of new chiral compounds requires development of new CSPs that can determine the ee yield of separated enantiomers.

\footnotetext{
${ }^{a}$ Charles University in Prague, Faculty of Science, Department of Organic Chemistry, Hlavova 8, 12843 Prague 2, Czech Republic.

E-mail: petr.milko@weizmann.ac.il, jana.roithova@natur.cuni.cz; Tel: +420-221-951-322

${ }^{b}$ Department of Chemistry \& Biochemistry, The University of Texas at Arlington, 700 Planetarium Pl., Arlington, TX 76019-0065, USA. E-mail: kschug@uta.edu; Tel: +1-817-272-3541

${ }^{c}$ Regional Centre of Advanced Technologies and Materials, Department of Analytical Chemistry, Faculty of Science, Palacky University, 17. listopadu 12, 77146 Olomouc, Czech Republic. E-mail: karel.lemr@upol.cz; Tel: +420-585-634-415 $\dagger$ Electronic supplementary information (ESI) available: NCI plots, NBO analysis and $x y z$ coordinates of the all optimized structures are posted. See DOI: 10.1039/ c3cp44444a

† Present address: Department of Chemical Research Support, Weizmann Institute of Science, Rehovot 76100, Israel. Tel: +972-8-934-6218.
}

However, the development of new CSPs is an expensive process. A new compound must be synthesized in a sufficient amount and bound to a solid support to create a chromatographic column. Therefore, it is desirable to find an inexpensive and efficient screening method that would identify promising candidates. Mass spectrometry (MS) is a technique that allows routine, fast, and cheap analysis of samples. This fact has encouraged the development of mass spectrometric tools for investigating chiral recognition in the last decade. ${ }^{4-13}$ Methods of computational chemistry are another suitable approach. They are widely used in drug discovery as a cheap screening method ${ }^{14-16}$ or in biochemistry in order to shed light on the functions of enzymes. ${ }^{17}$

Herein, we present a computational study, using density functional theory to investigate structure and stability of non-covalently bound complexes of $(8 S, 9 R)$-tert-butylcarbamoylquinine ( $t$ BuCQN), $N$-3,5-dinitrobenzoyl-( $S, R)$-leucine (DNB- $S / R$-Leu), and alkali cations in the gas phase (Fig. 1a). The $t$ BuCQN molecule belongs to a family of quinine (Cinchona alkaloid) derivatives that have been successfully used as CSPs (Fig. 1b) for the separation of enantiomers of chiral acids, particularly N-blocked amino acids. ${ }^{18-20}$ This system has also been extensively studied by HPLC and electrospray ionization-mass spectrometry (ESI-MS) methods. ${ }^{2,9,21}$ Both molecules are bound together via non-covalent binding modes (Fig. 1b). Formation of the alkali complexes was observed in ESI-MS experiments and preferential 
a)<smiles>[Y][NH+]1CC2CC(C=C)[C@H]([C@H]([2H])c3ccnc4ccc(OC)cc34)C2C1</smiles>
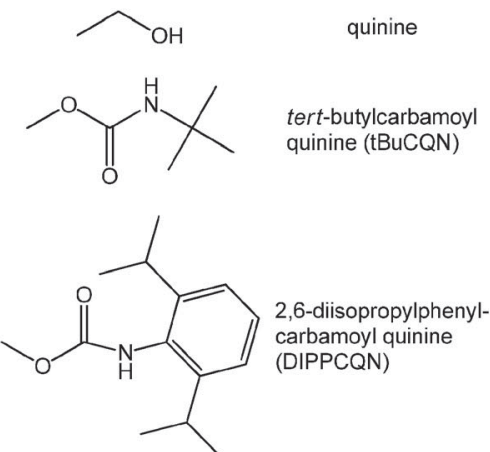



Fig. 1 (a) Representative Cinchona alkaloid quinine analogs, which have been investigated for use as chiral selectors. (b) Schematic representation of non-covalent binding interactions in the $t$ BuCQN.DNB-S/R-Leu complex. The interacting groups are highlighted in the same colour.

formation of a diastereoisomeric complex containing the $(S)$-optical isomer of DNB-Leu was shown to correlate well with HPLC results. The ESI-MS experiments further showed that enantioselectivity depends on the alkali metal involved in the complex. ${ }^{22}$ Thus, the best enantioselectivity was observed for the lithium complexes while efficiency of the enantioselectivity was lower for the sodium and potassium complexes. Therefore, special attention is paid to the understanding of the role and the placement of the alkali metal ion in the diastereomeric complexes to achieve different levels of enantioselectivity in this work.

\section{Methods}

All calculations were carried out using Gaussian 09 Revision A.02. ${ }^{23}$ The Becke-97D ${ }^{24}$ functional was used for geometry optimizations in conjunction with the $6-311++\mathrm{G}^{* *}$ basis set. $^{25-27}$ Density Fitting Basis Sets (DFBS) were generated using the automatic algorithm implemented in Gaussian 09 to reduce computational cost. $^{28,29}$ The B97D functional is a pure GGA functional based on the Becke-97 (ref. 30) functional and includes the second version of Grimme's empirical dispersion correction. The empirical dispersion correction term is given by

$$
E_{\mathrm{disp}}=-S_{6} \sum_{i=1}^{N_{\mathrm{at}}-1} \sum_{j=i+1}^{N_{\mathrm{at}}} \frac{C_{6}^{i j}}{R_{i j}{ }^{6}} f_{\mathrm{dmp}}\left(R_{i j}\right) .
$$

Here, $N_{\text {at }}$ corresponds to the number of atoms in the system, $C_{6}{ }^{i j}$ is the dispersion coefficient for atom pair $i j, s_{6}$ is a global scaling factor that depends only on the density functional used, $R_{i j}$ is the interatomic distance, and $f_{\mathrm{dmp}}$ is a damping function that decays at small $R$ fast enough to zero as the dispersion corrections are small between atoms below typical van der Waals distances and hence, "normal" bonds are not significantly affected by the correction. The $f_{\mathrm{dmp}}$ function is defined by

$$
f_{\mathrm{dmp}}=\frac{1}{1+\mathrm{e}^{-d\left(\frac{R_{i j}}{R_{\mathrm{r}}}-1\right)}}
$$

where $R_{\mathrm{r}}$ is the sum of atomic vdW radii and $d$ is a parameter.

The B97D functional was successfully tested for variety of systems including the rare gas and benzene dimers, transition metal complexes and so forth (for more details see ref. 24 and 31). A benchmark study was published by Á. Vázquez-Mayagoitia et $a l^{32}$ for benzene dimers and they showed that the B97D functional provides energies with errors less than $4.18 \mathrm{~kJ} \mathrm{~mol}^{-1}$, compared to $\operatorname{CCSD}(\mathrm{T})$. The B97D functional is thus a good compromise between the computational cost and the accuracy and was applied in different studies. ${ }^{33-35}$ Frequency calculations were done at the same level of theory to check the identity of all minima. Relative energies of the optimized structures were obtained by single-point energy calculations using the PBE0 functional (also denoted PBE1PBE) in conjunction with a $6-311++G^{* *}$ basis set and the "ultrafine" integration grid (i.e., a pruned $(99,590)$ grid). The PBE0 functional is a hybrid version of the PBE functional incorporating 25\% Hartree-Fock exchange. ${ }^{36-38}$ The relative energies are corrected for the dispersion effect by the third version of the Grimme empirical model. ${ }^{31}$ Partial atomic charges were obtained from Charge Model 5 (CM5) introduced by A. V. Marenich et al. ${ }^{39-43}$ that yields class IV charges derived from Hirshfeld population analysis. ${ }^{41}$ The NCIPLOT program, which was developed by Contreras-García et al., was used in an attempt to identify and visualize non-covalent interactions. ${ }^{44}$ It analyzes the reduced density gradient $s$ of the electron density $\rho$ obtained by the

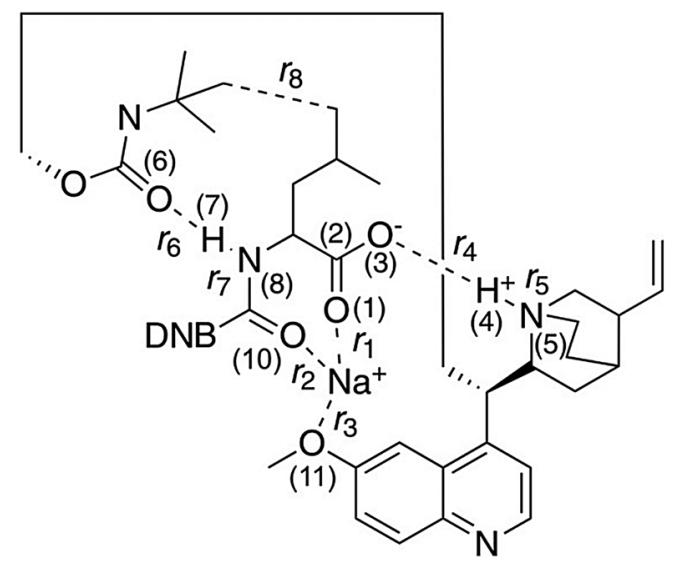

Scheme 1 Schematic representation of the [tBuCQN.DNB-S/R-Leu.Na $]^{+}$complex. 
(a)

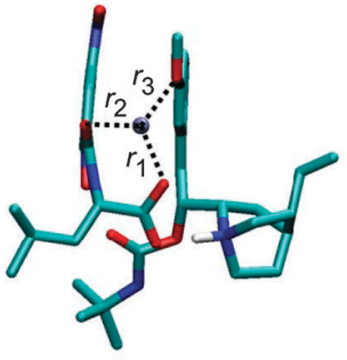

(b)

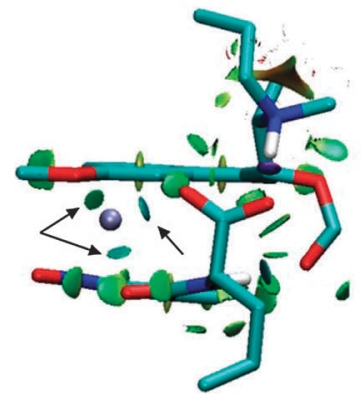



(c)



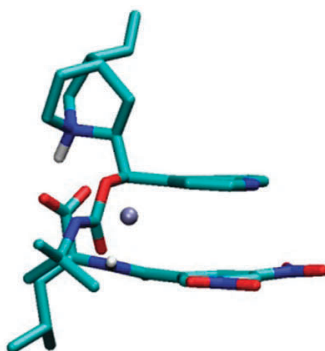

(d)

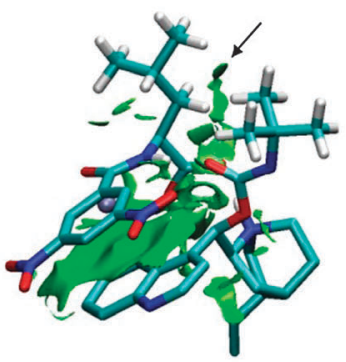

(e)

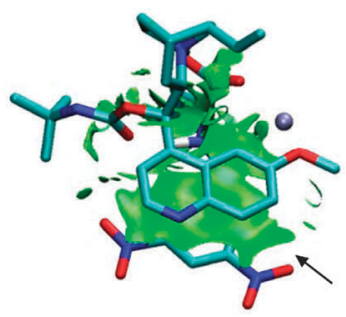

Fig. 2 The optimized structures of the $1_{s} \mathrm{Na}^{+}$complex (B97D/6-311++G**/DFBS). Hydrogen atoms were removed except for the important ones to improve the clarity of the pictures. Colour codes: light blue, white, red, blue, and ice-blue correspond to carbon, hydrogen, oxygen, nitrogen, and sodium, respectively. Colour codes of the isosurfaces: the weak-, the strong attractive-, and the strong repulsive interactions are in green, blue, and red, respectively. The black arrows indicate the isosurfaces discussed in the text.

single-point energy calculations at low densities that are related to the weakest interaction. Therefore, a density cutoff of $\rho<0.1$ a.u. was chosen as it includes the region of interest (see Fig. 3).

\section{Results}

Formation of singly charged complexes [ $t \mathrm{BuCQN} \cdot \mathrm{DNB}-S / R$ Leu $\cdot \mathrm{Na}]^{+}$was observed in previous ESI-MS experiments. ${ }^{2}$ Molecules are reasoned to be bound via four non-covalent interactions (Fig. 1b): (i) a Coulombic attraction of carboxylic oxygen $\mathrm{O}^{-}(3)$ (Scheme 1) of DNB-Leu and the protonated amine group $\mathrm{N}(5)-\mathrm{H}(4)^{+}$of $t \mathrm{BuCQN}$, (ii) a hydrogen bond between carbonyl of the carbamoyl group $\mathrm{O}(6)$ of $t$ BuCQN and $\mathrm{H}(7)$ of the amide group of DNB-Leu, (iii) the $\pi-\pi$ interaction ( $\pi$ stacking) of dinitrobenzoyl and 6-methoxy-quinolyl rings, and (iv) van der Waals interactions between the alkyl chains. The presence of sodium in the complexes raises a question about its location. The sodium atom can be rationally positioned at three potential binding sites in the complexes. Both molecules of the complexes have aromatic moieties that offer two binding modes for the sodium atom by a cation $-\pi$ interaction. ${ }^{45}$ The third possibility is an ionic bond between the sodium atom and the carboxylate group of DNB-Leu.

The crystal structure of a complex of $\beta$-chloro-tert-butylcarbamoylquinine and DNB- $S$-Leu was taken as a starting point for geometry optimizations. ${ }^{20}$ The original crystal structure was modified by replacing the chlorine atom with hydrogen and adding a sodium atom. It was found that a complex containing
Table 1 Relative energies and entropies of the optimized complexes (obtained at the PBE0/6-311++G**//B97D/6-311++G**/DFBS level of theory)

\begin{tabular}{lrc}
\hline $1_{S / R} \mathrm{X}^{+}$ & $\Delta_{\text {rel }} G^{298}\left(\mathrm{~kJ} \mathrm{~mol}^{-1}\right)$ & $\Delta_{\text {rel }} S^{298}\left(\mathrm{~J} \mathrm{~mol}^{-1} \mathrm{~K}^{-1}\right)$ \\
\hline $1_{S} \mathrm{Na}^{+}$ & 0.0 & 0.000 \\
$2_{S} \mathrm{Na}^{+}$ & 175.9 & 8.916 \\
$3_{S} \mathrm{Na}^{+}$ & 78.7 & 12.267 \\
$1_{R} \mathrm{Na}^{+}$ & 14.9 & 3.862 \\
$2_{R} \mathrm{Na}^{+}$ & 89.5 & 37.510 \\
$3_{R} \mathrm{Na}^{+}$ & 64.4 & 38.614 \\
$1_{S} \mathrm{Li}^{+}$ & 0.0 & 0.000 \\
$1_{R} \mathrm{Li}^{+}$ & 18.7 & -12.221 \\
$1_{S} \mathrm{~K}^{+}$ & 0.0 & 0.000 \\
$1_{R} \mathrm{~K}^{+}$ & 6.5 & 2.753 \\
\hline
\end{tabular}

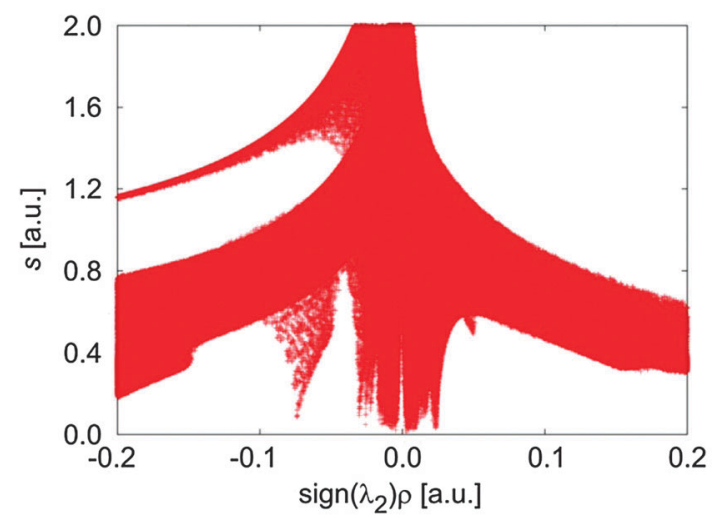

Fig. 3 Dependence of the reduced density gradient on the electron density multiplied by the sign of the second Hessian eigenvalue - so called $\mathrm{NCl}$ plot for ${ }_{s} \mathrm{Na}^{+}$. 
the $\mathrm{Na}^{+}-{ }^{-}$OOC pair $\left(1_{S} \mathrm{Na}^{+}\right.$; Fig. 2$)$ is the most stable one (Table 1) compared to the other complexes with the cation- $\pi$ interaction $\left(2{ }_{S} \mathrm{Na}^{+}\right.$and $3_{S} \mathrm{Na}^{+}$; Fig. 4 and 5$)$. The sodium cation in $1_{S} \mathrm{Na}^{+}$is coordinated in a pool of oxygen atoms (the amidic $\mathrm{O}(10)$, carbonyl $\mathrm{O}(1)$ of the DNB- $S$-Leu carboxylate group and the methoxy group $\mathrm{O}(11)$ of $t$ BuCQN, Scheme 1), as shown by the three attractive isosurfaces surrounding the sodium cation (Fig. 2b). The next attractive isosurface belongs to the strong Coulombic interaction of the $\mathrm{O}^{-}(3)-\mathrm{H}^{+}(4)$ pair. In the plot of the reduced density gradient versus the electron density multiplied by the sign of the second Hessian eigenvalue (so called NCI plot), a spike that corresponds to this interaction is significantly shifted to a negative value of the electron density with a peak at -0.074 a.u. (Fig. 3). The large shift indicates that the $\mathrm{O}^{-}(3)-\mathrm{H}^{+}(4)$ pair is not a purely ionic bond and has some covalent character. An isosurface of an attractive interaction is found for the next binding mode, the hydrogen bond (Fig. 2c) in $1_{S} \mathrm{Na}^{+}$. The weak attractive interaction is revealed between the two alkyl chains (Fig. 2d) and another low density surface is between the overlapping portion of the ring moieties related to the $\pi-\pi$ interaction (Fig. 2e). The $\pi-\pi$ interaction is strong as indicated by the short ring-ring distance $r_{9}$ (3.276 A, Table 2). For comparison, the calculated distances for benzene- or benzenepyridine sandwich dimers are in the order of 3.8-3.9 $\AA^{46,47}$ Placing the sodium atom on the top of the 6-methoxy-quinolyl ring leads to a considerably less stable complex $2_{S} \mathrm{Na}^{+}$(compared to $\left.1_{S} \mathrm{Na}^{+}\right)$. The sodium atom is positioned above the heterocyclic moiety and is bound by the cation $-\pi$ interaction (Fig. $4 \mathrm{~b}$ and c). The proton bound to the nitrogen atom of the bicyclic moiety and forming the $\mathrm{O}^{-}(3)-\mathrm{H}^{+}(4)$ pair spontaneously migrates to the carboxylic group during the optimization (Fig. 4c). The $2_{S} \mathrm{Na}^{+}$ complex thus contains another hydrogen bond instead of the

Table 2 Monitored distances $(\AA)$ for the most stable structures of [tBuCQN-DNB-S/R-Leu.X] $]^{+}$complexes, where $X=N a$, Li, and $K$

\begin{tabular}{|c|c|c|c|c|c|c|c|c|c|c|c|}
\hline $1_{S / R} \mathrm{X}^{+}$ & $r_{1}$ & $r_{2}$ & $r_{3}$ & $r_{4}$ & $r_{5}$ & $r_{4}+r_{5}$ & $r_{6}$ & $r_{7}$ & $r_{6}+r_{7}$ & $r_{8}$ & $r_{9}$ \\
\hline $1_{S} \mathrm{Na}^{+}$ & 2.247 & 2.279 & 2.416 & 1.531 & 1.093 & 2.624 & 1.839 & 1.023 & 2.862 & 4.257 & 3.276 \\
\hline $2{ }_{S} \mathrm{Na}^{+}$ & & & & 1.026 & 1.698 & 2.724 & 1.926 & 1.018 & 2.944 & 5.190 & 3.282 \\
\hline $3_{S} \mathrm{Na}^{+}$ & & 2.243 & 2.515 & 1.498 & 1.114 & 2.612 & 1.940 & 1.024 & 2.964 & 5.848 & - \\
\hline $1_{R} \mathrm{Na}^{+}$ & 2.240 & 2.250 & 2.433 & 1.517 & 1.096 & 2.613 & 1.869 & 1.020 & 2.889 & 5.907 & 3.268 \\
\hline $2{ }_{R} \mathrm{Na}^{+}$ & 2.435 & 2.271 & 2.380 & - & - & - & 1.845 & 1.027 & 2.872 & 3.612 & 3.301 \\
\hline $3_{R} \mathrm{Na}^{+}$ & 2.424 & 2.403 & 2.388 & 1.501 & 1.000 & 2.501 & - & - & - & 3.728 & - \\
\hline $1_{S} \mathrm{Li}^{+}$ & 1.885 & 1.911 & 2.088 & 1.539 & 1.088 & 2.627 & 1.859 & 1.021 & 2.880 & 4.428 & 3.267 \\
\hline $1_{R} \mathrm{Li}^{+}$ & 1.869 & 1.898 & 2.093 & 1.543 & 1.087 & 2.630 & 1.909 & 1.019 & 2.928 & 5.952 & 3.270 \\
\hline $1_{S} \mathrm{~K}^{+}$ & 2.601 & 2.594 & 2.893 & 1.539 & 1.069 & 2.608 & 1.943 & 1.018 & 2.961 & 4.052 & 3.270 \\
\hline $1_{R} \mathrm{~K}^{+}$ & 2.745 & 2.697 & 2.861 & 1.499 & 1.091 & 2.590 & 1.838 & 1.017 & 2.855 & 4.432 & 3.289 \\
\hline
\end{tabular}

(a)

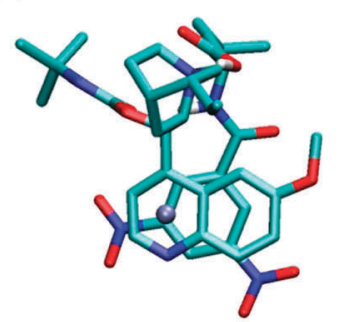

(c)

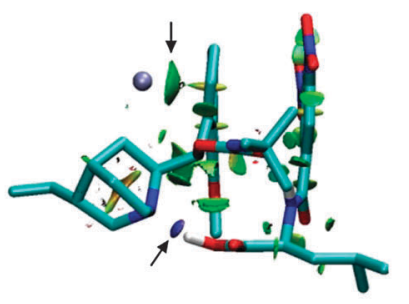



(d)



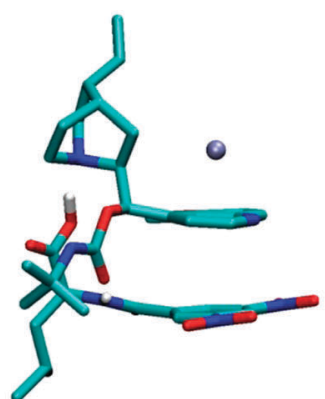

(e)

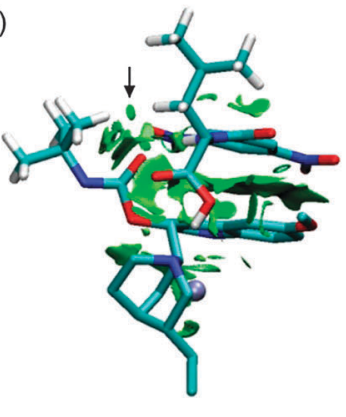

(b)

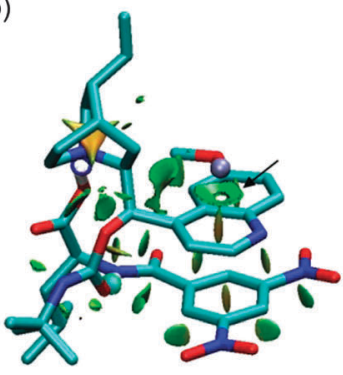

(f)

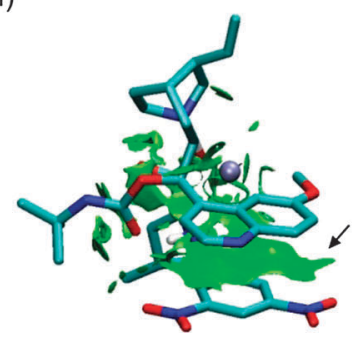

Fig. 4 The optimized structure of the ${ }_{S} \mathrm{Na}^{+}$complex (B97D/6-311++G**/DFBS). Hydrogen atoms were removed except for the important ones to improve the clarity of the pictures. Colour codes: light blue, white, red, blue, and ice-blue correspond to carbon, hydrogen, oxygen, nitrogen, and sodium, respectively. Colour codes of the isosurfaces: the weak-, the strong attractive-, and the strong repulsive interactions are in green, blue, and red, respectively. The black arrows indicate the isosurfaces discussed in the text. 
Table 3 Selected group CM5 partial charges of complexes. X stands for $\mathrm{Na}$, $\mathrm{Li}$, and $\mathrm{K}$

\begin{tabular}{|c|c|c|c|c|c|c|c|}
\hline $1_{S / R} \mathrm{X}^{+}$ & $\mathrm{X}$ & $\begin{array}{l}\text { COO } \\
\text { (DNB-Leu) }\end{array}$ & $\begin{array}{l}\text { CO } \\
\text { (DNB- } \\
\text { Leu) }\end{array}$ & $\begin{array}{l}\mathrm{CT}^{a} \\
\text { (DNB- } \\
\text { Leu) }\end{array}$ & $\begin{array}{l}\mathrm{OCH}_{3} \\
(t \mathrm{BuCQN})\end{array}$ & $\begin{array}{l}\text { The bicyclic } \\
\text { residue } \\
(t \mathrm{BuCQN})\end{array}$ & $\begin{array}{l}\mathrm{CT}^{a} \\
(t \mathrm{BuCQN})\end{array}$ \\
\hline $1_{S} \mathrm{Na}^{+}$ & 0.76 & -0.61 & -0.16 & 0.34 & -0.03 & 0.69 & -0.10 \\
\hline $2_{S} \mathrm{Na}^{+}$ & 0.75 & -0.13 & -0.06 & -0.10 & 0.02 & 0.20 & 0.35 \\
\hline $3_{S} \mathrm{Na}^{+}$ & 0.69 & -0.54 & -0.14 & $=0.42$ & 0.01 & 0.65 & -0.11 \\
\hline $1_{R} \mathrm{Na}^{+}$ & 0.76 & -0.64 & -0.07 & 0.43 & -0.09 & 0.68 & -0.19 \\
\hline $2{ }_{R} \mathrm{Na}^{+}$ & 0.77 & -0.05 & -0.14 & 0.96 & -0.07 & 0.08 & -0.73 \\
\hline $3_{R} \mathrm{Na}^{+}$ & 0.73 & -0.64 & -0.10 & 0.43 & -0.03 & 0.67 & -0.16 \\
\hline $1_{S} \mathrm{Li}^{+}$ & 0.60 & -0.58 & -0.14 & 0.42 & 0.01 & 0.70 & -0.02 \\
\hline $1_{R} \mathrm{Li}^{+}$ & 0.76 & -0.69 & -0.08 & 0.41 & -0.08 & 0.67 & -0.17 \\
\hline $1_{S} \mathrm{~K}^{+}$ & 0.86 & -0.64 & -0.17 & 0.29 & -0.08 & 0.71 & -0.15 \\
\hline $1_{R} \mathrm{~K}^{+}$ & 0.84 & -0.68 & -0.14 & 0.28 & -0.03 & 0.69 & -0.11 \\
\hline
\end{tabular}

Coulombic attraction. Compared to the $\mathrm{O}(6) \cdots \mathrm{H}(7)-\mathrm{N}(8)$ hydrogen bond, this $\mathrm{N}(5) \cdots \mathrm{H}(4)-\mathrm{O}(3)$ hydrogen bond is very strong (Fig. $4 \mathrm{c}$ and d) with a spike at the reduced density gradient plot at -0.057 a.u. (see Fig. S1, ESI + ). Furthermore, the charge distribution is influenced and the opposite direction of charge transfer is observed in that positive charge is on $t \mathrm{BuCQN}$ and DNB-S-Leu becomes negative (Table 3). Removing the sodium atom from the bonding distance of the carboxylic group of DNB-S-Leu has thus a significant impact on the entire complex. The $\mathrm{O}(6) \cdots \mathrm{H}(7)-\mathrm{N}(8)$ hydrogen bond is weaker compared to $1_{S} \mathrm{Na}^{+}$and the $r_{8}$ distance is elongated that results only in a weak interaction of two hydrogen atoms (Fig. 4e) with respect to $1_{S} \mathrm{Na}^{+}$. Interestingly, no significant impact on the $\pi-\pi$ interaction occurs (Fig. 4f). In the last tested isomer $3_{S} \mathrm{Na}^{+}$, the sodium cation is placed above the dinitrobenzoyl moiety. Nevertheless, the sodium cation is attracted by the oxygen atom $\mathrm{O}(10)$ of the amidic carbonyl during the optimization. In the final structure, it is coordinated to the oxygen atom $\mathrm{O}(10)$ of the amidic carbonyl via a strongly attractive interaction (Fig. 5b) and to the methoxy group and the $\pi$ system of the quinolyl moiety (Fig. 5e). The $\mathrm{O}^{-}(3)-\mathrm{H}^{+}(4)$ pair has more covalent character than in $1_{S} \mathrm{Na}^{+}\left(\operatorname{sign}\left(\lambda_{2}\right) \rho=-0.082\right.$; see Fig. S2, ESI $\left.\dagger\right)$ and the $\mathrm{O}(6) \cdots \mathrm{H}(7)-\mathrm{N}(8)$ hydrogen bond (Fig. 5c) is weaker than in $1_{S} \mathrm{Na}^{+}$. The alkyl groups are put apart compared to ${ }_{1} \mathrm{Na}^{+}$with the large $r_{8}$ distance and similar to $2_{S} \mathrm{Na}^{+}$there is a weak interaction of two hydrogen atoms of the alkyl groups (Fig. $5 \mathrm{~d}$ ). The $\pi-\pi$ interaction of the two aromatic rings is diminished (Fig. 5e). Hence hereafter, the most stable isomer $1_{S} \mathrm{Na}^{+}$represents a reference for the following discussion of additional results.

Replacing of the $(S)$ optical isomer of DNB-Leu for the $(R)$ isomer leads to complexes in which at least one binding mode is missing. It can be intuitively expected that switching of the hydrogen- and the alkyl positions at the chiral center of DNB-Leu will have an effect on the stability of $[t$ BuCQN.DNBLeu-Na $]^{+}$. Indeed, the $1_{R} \mathrm{Na}^{+}$complex (Fig. 6) is found to be energetically preferred over $2_{R} \mathrm{Na}^{+}$and $3_{R} \mathrm{Na}^{+}$. The $r_{1}, r_{2}$ and $r_{3}$ distances are shortened in $1_{R} \mathrm{Na}^{+}$compared to $1_{S} \mathrm{Na}^{+}$. The strength of the interaction in the $\mathrm{O}^{-}(3)-\mathrm{H}^{+}(4)$ pair is increased, which is reflected by a shortening of $r_{4}$ and also by the increased (a)

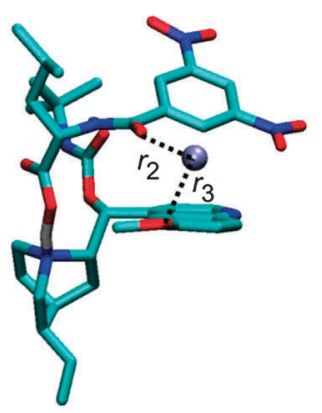

(b)

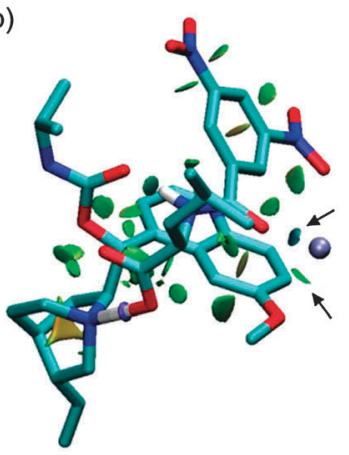

(c)

(d)



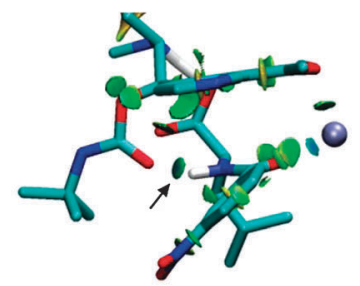

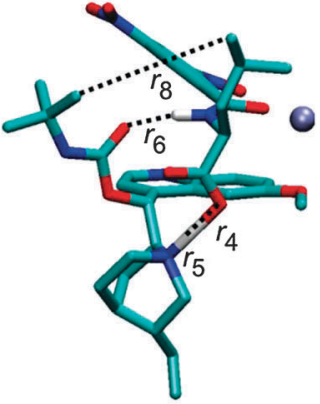

(e)



Fig. 5 The optimized structure of the ${ }_{3} \mathrm{Na}^{+}$complex (B97D/6-311++G**/ DFBS). Hydrogen atoms were removed except for the important ones to improve the clarity of the pictures. Colour codes: light blue, white, red, blue, and ice-blue correspond to carbon, hydrogen, oxygen, nitrogen, and sodium, respectively. Colour codes of the isosurfaces: the weak-, the strong attractive-, and the strong repulsive interactions are in green, blue, and red, respectively. The black arrows indicate the isosurfaces discussed in the text.

value of $\operatorname{sign}\left(\lambda_{2}\right) \rho$, which is -0.077 a.u. (Fig. 6c, Fig. S3, ESI + ). The stronger hydrogen bond is found in $1_{R} \mathrm{Na}^{+}$than in $1_{S} \mathrm{Na}^{+}$as shown by shorter $r_{6}$ in $1_{R} \mathrm{Na}^{+}$(Fig. 6c). In contrast, interaction of the two alkyl chains diminishes in $1_{R} \mathrm{Na}^{+}$compared to $1_{S} \mathrm{Na}^{+}$ as indicated by the large value of $r_{8}$ and also the missing isosurface of the weak interaction (Fig. 6d). The position of the dinitrobenzoyl ring is similar in $1_{R} \mathrm{Na}^{+}$and $1_{S} \mathrm{Na}^{+}$and there is only a small difference in $r_{9}$ in these two complexes (Fig. 6e). The next considered complex contains the carboxylic group rotated away from the close distance of the protonated nitrogen. The rotation brings the alkyl groups closer together and results in a stronger van der Waals interaction. However, during the optimization, the Coulombic attraction prevailed over the vdW interaction and the optimization led again to the $1_{R} \mathrm{Na}^{+}$isomer. The way to prevent this result is to neutralize the carboxylate group. Optimization of the suggested structure provided a stable 
(a)
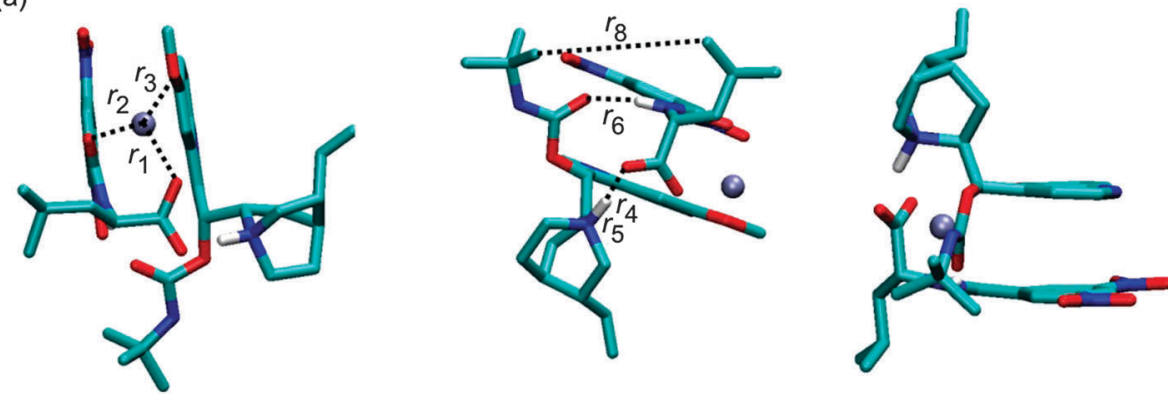

(b)

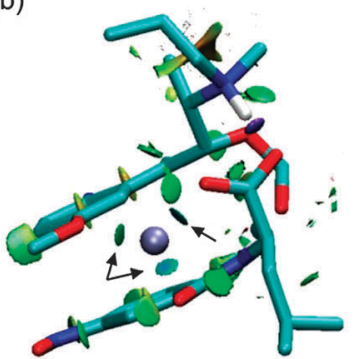

(c)

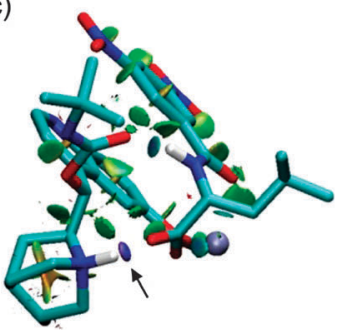

(d)



(e)

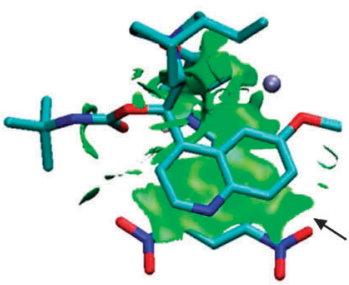

Fig. 6 The optimized structure of the $1_{R} \mathrm{Na}^{+}$complex (B97D/6-311++G**/DFBS). Hydrogen atoms were removed except for the important ones to improve the clarity of the pictures. Colour codes: light blue, white, red, blue, and ice-blue correspond to carbon, hydrogen, oxygen, nitrogen, and sodium, respectively. Colour codes of the isosurfaces: the weak-, the strong attractive-, and the strong repulsive interactions are in green, blue, and red, respectively. The black arrows indicate the isosurfaces discussed in the text.

isomer $2_{R} \mathrm{Na}^{+}$(Fig. 7). The sodium atom remains coordinated by three oxygen atoms similarly to $1_{S} \mathrm{Na}^{+}$(Fig. 7b) but the carboxylic group is neutral, which leads to a missing spike related to the $\mathrm{O}^{-}(3)-\mathrm{H}^{+}(4)$ pair interaction in the NCI plot (Fig. S4, ESI $\dagger$ ). The $\mathrm{O}(6) \cdots \mathrm{H}(7)-\mathrm{N}(8)$ bond (Fig. 7c) is slightly affected as $r_{6}$ is elongated. Interaction of the alkyl chains (Fig. 7d) increases with respect to $1_{S} \mathrm{Na}^{+}$while the $\pi-\pi$ interaction (Fig. 7e) is weaker than in $1_{S} \mathrm{Na}^{+}$. Therefore, the Coulombic interaction of the $\mathrm{O}^{-}(3)-\mathrm{H}^{+}(4)$ pair has a significant impact on the overall stability of $[t \text { BuCQN.DNB-Leu } \cdot \mathrm{Na}]^{+}$. At the last, an isomer, $3_{R} \mathrm{Na}^{+}$, with the broken $\mathrm{O}(6) \cdots \mathrm{H}(7)-\mathrm{N}(8)$ hydrogen bond and the $\pi-\pi$ interaction is considered (Fig. 8). Again there is a weakening of an interaction of the sodium cation with the oxygen atoms compared to the binding situation in $1_{S} \mathrm{Na}^{+}$ (Fig. 8b). The $3_{R} \mathrm{Na}^{+}$isomer contains a stronger interaction in the $\mathrm{O}^{-}(3)-\mathrm{H}^{+}(4)$ pair than in $1_{S} \mathrm{Na}^{+}$(Fig. 8b and Fig. S5, ESI $\dagger$ ) and also significantly shorter $r_{8}$ (Fig. 8c). The missing hydrogen bond and the $\pi-\pi$ interaction lead to an isomer with an energetic stability between $1_{R} \mathrm{Na}^{+}$and $2_{R} \mathrm{Na}^{+}$. Clearly, both binding modes contribute to the stability of $[t \mathrm{BuCQN} \cdot \mathrm{DNB}-\mathrm{Leu} \cdot \mathrm{Na}]^{+}$less than the Coulomb interaction.

Finally, we will address the question, whether the ion-pair $\left(\mathrm{O}^{-}(3)-\mathrm{H}^{+}(4)\right)$ forms of the complexes $1_{S / R} \mathrm{Na}^{+}$are really energetically preferred to their neutral counter-parts what might sound counterintuitive in the gas phase. The relaxed potential scans of a proton migration from $\mathrm{N}(5)$ to $\mathrm{O}^{-}(3)$ were done for both isomers to answer this question. The scans show that the proton migration from the nitrogen atom to the oxygen atom is continuously endothermic (see Fig. S14-S19, ESI + ). The relative energies of the neutral complexes are more than $18.0 \mathrm{~kJ} \mathrm{~mol}^{-1}$ above the energies of the ion-pair complexes. Hence, the spontaneous proton migration from $\mathrm{O}^{-}(3)$ to $\mathrm{N}(5)$ occurred during the full optimization of the complexes and the ionic form of the $\mathrm{O}(3)-\mathrm{H}(4)$ pair is preferred in the gas phase.

In summary, $1_{S} \mathrm{Na}^{+}$and $1_{R} \mathrm{Na}^{+}$are identified to be the most stable diastereoisomers and will be considered in the following discussion. Analysis of the stabilities of all computed isomers allows us to order all binding modes according to their contribution to the stabilization of the $[t \text { BuCQN.DNB-Leu.Na }]^{+}$complexes in the following order: the Coulomb interaction $>$ the hydrogen bond $\sim$ the $\pi-\pi$ interaction $>$ van der Waals interaction. While the van der Waals interactions provide the smallest contribution to the stabilization, they make a decisive difference between the diastereoisomeric complexes and are responsible for the large potential of $t \mathrm{BuCQN}$ for the enantioselective recognition.

\section{The effect of a counter ion}

It was shown experimentally that changing a counter ion has a significant influence on the energy stabilities of the studied diastereoisomers. $^{22}$ In the case of the lithium counter ions, enhancement in the separation ability of $t$ BuCQN was observed compared to the sodium cations while using the potassium counter ions had an opposite effect. The $1_{S} \mathrm{Na}^{+}$and $1_{R} \mathrm{Na}^{+}$complexes are reoptimized with the $\mathrm{Li}$ and $\mathrm{K}$ counter ions in an attempt to see the relevant differences (see optimized structures in Fig. S6-S9, ESI $\dagger$ ). The $r_{1}, r_{2}$, and $r_{3}$ bond lengths are shortened in the lithium complexes compared to the sodium ones, especially in $1_{R} \mathrm{Li}^{+}$. The stronger interaction of $\mathrm{Li}^{+}$with $\mathrm{O}(1), \mathrm{O}(10)$, and $\mathrm{O}(11)$ is also confirmed by the respective isosurfaces that have a deeper blue colour (see Fig. 2b, 6b, Fig. S6b and S7b, ESI $\dagger$ ). 
(a)

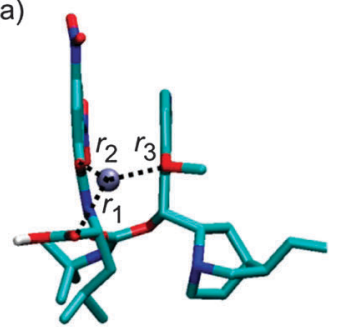

(b)

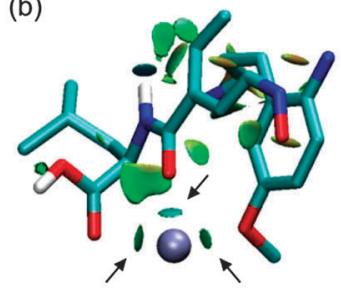

(d)





(c)



(e)

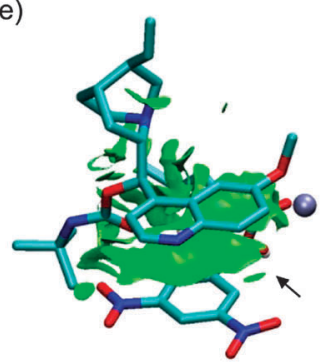

Fig. 7 The optimized structure of the $2{ }_{R} \mathrm{Na}^{+}$complex (B97D/6-311++G**/ DFBS). Hydrogen atoms were removed except for the important ones to improve the clarity of the pictures. Colour codes: light blue, white, red, blue, and ice-blue correspond to carbon, hydrogen, oxygen, nitrogen, and sodium, respectively. Colour codes of the isosurfaces: the weak-, the strong attractive-, and the strong repulsive interactions are in green, blue, and red, respectively. The black arrows indicate the isosurfaces discussed in the text.

The $\mathrm{O}^{-}(3)-\mathrm{H}^{+}(4)$ pair is affected slightly by the $\mathrm{Na} \rightarrow \mathrm{Li}$ exchange as well as the $\mathrm{O}(6) \cdots \mathrm{H}(7)-\mathrm{N}(8)$ hydrogen bond and the $\pi-\pi$ interaction. The more electronegative lithium absorbs more of the negative charge from the $\mathrm{COO}^{-}$group and it causes a lowering of the charge transfer from DNB-Leu to $t$ BuCQN (Fig. 9). Consequently, the $\mathrm{H}(4)$ atom is bound tighter to $\mathrm{N}(5)$ and the $\mathrm{O}^{-}(3)-\mathrm{H}^{+}(4)$ ionic interaction is weaker in $1_{S / R} \mathrm{Li}^{+}$than in $1_{S / R} \mathrm{Na}^{+}$. The weakening is even more pronounced for $1_{R} \mathrm{Li}^{+}$ that is an opposite situation with respect to $1_{S / R} \mathrm{Na}^{+}$in which the $\mathrm{O}^{-}(3)-\mathrm{H}^{+}(4)$ is stronger in $1_{R} \mathrm{Na}^{+}$than in $1_{S} \mathrm{Na}^{+}$. This can be also seen from a shift of the related spikes in the NCI plots $\left(\left(\operatorname{sign}\left(\lambda_{2}\right) \rho\right.\right.$ is -0.072 and -0.071 for $1_{S} \mathrm{Li}^{+}$and $1_{R} \mathrm{Li}^{+}$, respectively; see Fig. S10 and S11, ESI $\dagger$ ). The impact of the $\mathrm{Na} \rightarrow \mathrm{Li}$ exchange on the interaction of the two alkyl groups is small. The $r_{8}$ distance is elongated in the Li complexes with respect to the sodium ones.

Similarly to $1_{R} \mathrm{Na}^{+}$, isosurfaces relating to the interaction of the two alkyl groups are not present in $1_{R} \mathrm{Li}^{+}$. Thus, the enantioselectivity is enhanced by the weakening of the $\mathrm{O}^{-}(3)-$ $\mathrm{H}^{+}(4)$ pair in $1_{S} \mathrm{Li}^{+}$and $1_{R} \mathrm{Li}^{+}$compared to those in $1_{S} \mathrm{Na}^{+}$and $1_{R} \mathrm{Na}^{+}$in that the weakening is more pronounced for $1_{R} \mathrm{Li}^{+}$.

(a)
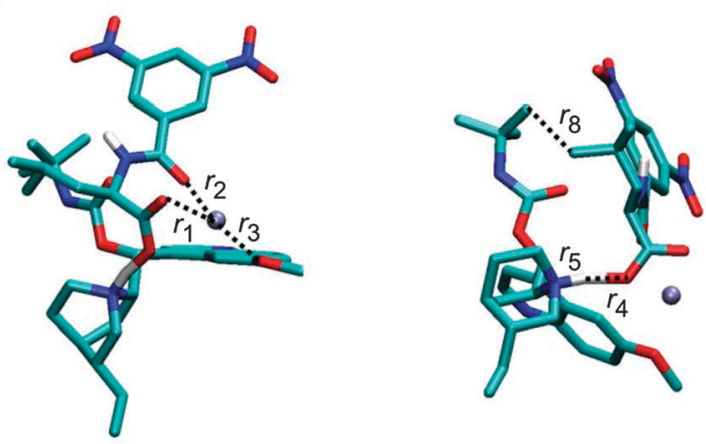

(b)

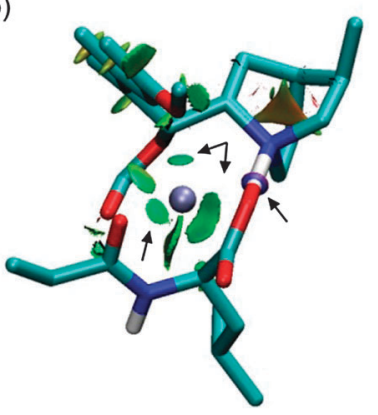

(c)

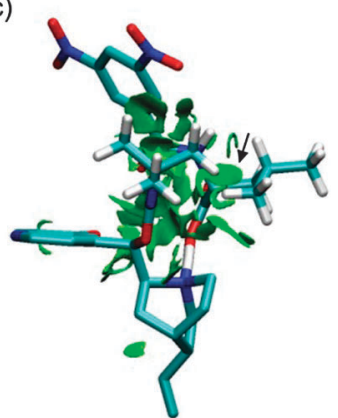

Fig. 8 The optimized structure of the $3_{R} \mathrm{Na}^{+}$complex (B97D/6-311++G**/ DFBS). Hydrogen atoms were removed except for the important ones to improve the clarity of pictures. Colour codes: light blue, white, red, blue, and ice-blue correspond to carbon, hydrogen, oxygen, nitrogen, and sodium, respectively. Colour codes of the isosurfaces: the weak-, the strong attractive-, and the strong repulsive interactions are in green, blue, and red, respectively. The black arrows indicate the isosurfaces discussed in the text.

In the case of the potassium complexes (Fig. S8 and S9, ESI + ), all three distances, $r_{1}, r_{2}$, and $r_{3}$, are larger compared to the sodium and lithium complexes. The $\mathrm{O}^{-}(3)-\mathrm{H}^{+}(4)$ pair interaction is weakened in $1_{S} \mathrm{~K}^{+}$with respect to $1_{S} \mathrm{Na}^{+}$, which is also indicated by the value of $\operatorname{sign}\left(\lambda_{2}\right) \rho=-0.064$ (Fig. S12, ESI $\dagger$ ) of the related spike in the NCI plot. The stronger ionic pair interaction is found in $1_{R} \mathrm{~K}^{+}$than in $1_{S} \mathrm{~K}^{+}$and $1_{S} \mathrm{Na}^{+}$ $\left(\operatorname{sign}\left(\lambda_{2}\right) \rho=-0.076\right.$; Fig. S13, ESI $\left.\dagger\right)$. The lower electronegativity of $\mathrm{K}$ than $\mathrm{Na}$ and Li causes increasing charge transfer between the molecules with nearly single positive charge on $\mathrm{K}$ (Fig. 9). The Na $\rightarrow \mathrm{K}$ exchange leads to the moderate weakening of the hydrogen bond in $1_{S} \mathrm{~K}^{+}$compared to $1_{S} \mathrm{Na}^{+}$while strength of the hydrogen bond increases in its diastereoisomer $1_{R} \mathrm{~K}^{+}$ with respect to $1_{S} \mathrm{~K}^{+}$. On the other hand, the $r_{8}$ distance is shortened in $1_{S} \mathrm{~K}^{+}$comparing with $1_{S} \mathrm{Na}^{+}$and also $1_{R} \mathrm{~K}^{+}$. The value of $r_{9}$ then indicates that the $\pi-\pi$ interaction is influenced only slightly by the $\mathrm{Na} \rightarrow \mathrm{K}$ exchange. The potassium counter ion makes the most important binding modes in the complex weaker in $1_{S} \mathrm{~K}^{+}$but stronger in $1_{R} \mathrm{~K}^{+}$similarly as in the sodium complexes. Consequently, the relative difference in the stabilities of $1_{S} \mathrm{~K}^{+}$and $1_{R} \mathrm{~K}^{+}$is smaller compared to the $1_{S} \mathrm{Na}^{+} /$ $1_{R} \mathrm{Na}^{+}$pair and hence the enantioselectivity is poor using the potassium counter ion.

In summary, exchanging of the counter ion has an impact on three binding modes in each particular diastereoisomer. Only the $\pi-\pi$ interaction does not show any significant 


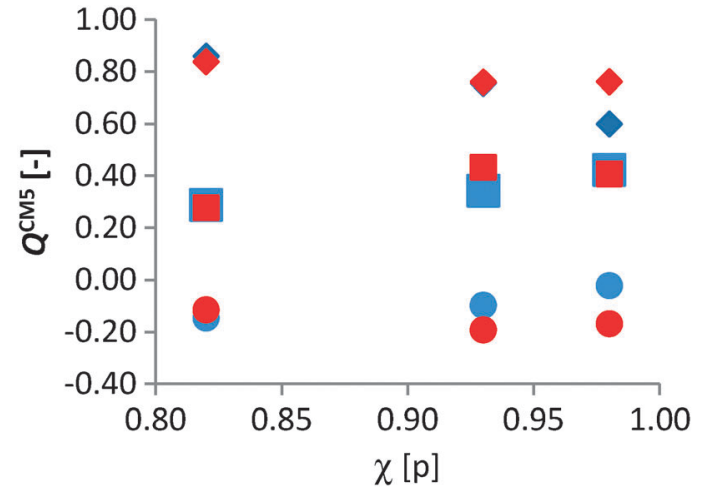

Fig. 9 Dependence of charge transfers on electronegativity of the alkali metals $(\mathrm{X}=\mathrm{Li}, \mathrm{Na}$, and $\mathrm{K})$. The blue colour corresponds to [tBuCQN.DNB-S-Leu.X $]^{+}$and the red colour corresponds to [tBuCQN.DNB-R-Leu-X] $]^{+}$. Diamond is a partial charge of the alkali ion, cube is the amount of the charge transfer from DNB$S / R$-Leu, and circle is the amount of the charge transfer to tBuCQN.

differences with the exchanging of the counter ions and remains invariant to this.

\section{Conclusions}

The presented study shows a lower stability of the $[t$ BuCQN.DNB$R$-Leu. Na $]^{+}$complex in comparison with its diastereoisomer $[t \text { BuCQN-DNB- } S \text {-Leu.Na }]^{+}$in the gas phase mainly due to a weaker van der Waals interaction of the alkyl chains in $[t \text { BuCQN} \cdot \mathrm{DNB}-\mathrm{R} \text {-Leu } \cdot \mathrm{Na}]^{+}$. Furthermore, the $\mathrm{O}^{-}(3)-\mathrm{H}^{+}(4)$ pair interaction is identified as the most stabilizing binding mode in $[t \mathrm{BuCQN} \cdot \mathrm{DNB}-S / R \text {-Leu.Na }]^{+}$complexes followed by the other binding modes: a hydrogen bond and the $\pi-\pi$ interaction. Replacing of the sodium cation with the more electronegative lithium cation causes a weakening of the $\mathrm{O}^{-}(3)-\mathrm{H}^{+}(4)$ pair interaction, the hydrogen bond and also van der Waals interaction. The weakening of the binding modes is more pronounced in $[t \mathrm{BuCQN} \cdot \mathrm{DNB}-R-\mathrm{Leu} \cdot \mathrm{Li}]^{+}$than in $[t \mathrm{BuCQN} \cdot \mathrm{DNB}-$ $S$-Leu $\cdot \mathrm{Li}]^{+}$. Hence, the energy difference between these two diastereoisomers is larger than for the sodium variants and thus the lithium ions are more suitable for the chiral recognition experiments. In contrast, the strength of the ionic pair interaction and the hydrogen bond is smaller in $[t \text { BuCQN-DNB- } S \text {-Leu- } \mathrm{K}]^{+}$than in $[t \text { BuCQN-DNB- } R \text {-Leu-K }]^{+}$. Therefore, the enantioselectivity is poor for the potassium complexes, despite the stronger vdW interaction in the potassium complexes. According to the work presented here, lithium appears to be the best counter ion in the chiral recognition within the $[t \mathrm{BuCQN} \cdots \mathrm{DNB}-S / R \text {-Leu } \cdots \mathrm{X}]^{+}$ $(\mathrm{X}=\mathrm{Li}, \mathrm{Na}, \mathrm{K}$ ) complexes, and this finding is consistent with the experimental mass spectrometry determinations.

\section{Acknowledgements}

The authors thank the late Dr habil. Detlef Schröder for providing computational time on the Horizoms computer cluster. This work was supported by the Ministry of Education of the Czech Republic (MSM0021620857). KAS acknowledges support from the National Science Foundation (CHE-0846310).
KL acknowledges support from Operational Program Research and Development for Innovations - European Regional Development Fund (project CZ.1.05/2.1.00/03.0058) and from the Czech Science Foundation (project P206/12/1150).

\section{Notes and references}

1 A. M. Rouhi, Chem. Eng. News, 2004, 82, 47.

2 K. A. Schug, P. Fryčák, N. M. Maier and W. Lindner, Anal. Chem., 2005, 77, 3660.

3 Y. Okamoto and T. Ikai, Chem. Soc. Rev., 2008, 37, 2593.

4 W. A. Tao and R. G. Cooks, Anal. Chem., 2003, 75, 25A.

5 S. Ahn, J. Ramirez, G. Grigorean and C. B. Lebrilla, J. Am. Soc. Mass Spectrom., 2001, 12, 278.

6 J. L. Seymour, F. Tureček, A. V. Malkov and P. Kočovský, J. Mass Spectrom., 2004, 39, 1044.

7 F. Gasparrini, M. Pierini, C. Villani, A. Filippi and M. Speranza, J. Am. Chem. Soc., 2008, 130, 522.

8 V. Ranc, V. Havlicek, P. Bednar and K. Lemr, Int. J. Mass Spectrom., 2009, 280, 213.

9 D. Scuderi, P. Maitre, F. Rondino, K. Le Barbu-Debus, V. Lepére and A. Zehnacker-Rentien, J. Phys. Chem. A, 2010, 114, 3306.

10 D. Scuderi, K. Le Barbu-Debus and A. Zehnacker, Phys. Chem. Chem. Phys., 2011, 13, 17916.

11 K. A. Schug and W. Lindner, J. Sep. Sci., 2005, 28, 1932.

12 K. A. Schug, Comb. Chem. High Throughput Screening, 2007, 10, 301.

13 A. Zehnacker, Chiral Recognition in the Gas Phase, Taylor \& Francis/CRC Press, 2010, p. 181.

14 M. Bollini, R. A. Domaoal, V. V. Thakur, R. Gallardo-Macias, K. A. Spasov, K. S. Anderson and W. L. Jorgensen, J. Med. Chem., 2011, 54, 8582.

15 A. Czarna, B. Beck, S. Srivastava, G. M. Popowicz, S. Wolf, Y. Huang, M. Bista, T. A. Holak and A. Dömling, Angew. Chem., Int. Ed., 2010, 49, 5352.

16 E. Laine, C. Goncalves, J. C. Karst, A. Lesnard, S. Rault, W. J. Tang, T. E. Malliavin, D. Ladant and A. Blondel, Proc. Natl. Acad. Sci. U. S. A., 2010, 107, 11277.

17 T. C. Bruice, Chem. Rev., 2006, 106, 3119.

18 N. M. Maier, P. Franco and W. Lindner, J. Chromatogr., A, 2001, 906, 3.

19 M. Lämmerhofer and W. Lindner, J. Chromatogr., A, 1996, 741, 33.

20 N. M. Maier, L. Nicoletti, M. Lämmerhofer and W. Lindner, Chirality, 1999, 11, 522.

21 N. M. Maier, S. Schefzick, G. M. Lombardo, M. Feliz, K. Rissanen, W. Lindner and K. B. Lipkowitz, J. Am. Chem. Soc., 2002, 124, 8611.

22 K. A. Schug, N. M. Maier and W. Linder, Chem. Commun., 2006, 414.

23 M. J. Frisch, G. W. Trucks, H. B. Schlegel, G. E. Scuseria, M. A. Robb, J. R. Cheeseman, G. Scalmani, V. Barone, B. Mennucci, G. A. Petersson, H. Nakatsuji, M. Caricato, X. Li, H. P. Hratchian, A. F. Izmaylov, J. Bloino, G. Zheng, J. L. Sonnenberg, M. Hada, M. Ehara, K. Toyota, R. Fukuda, 
J. Hasegawa, M. Ishida, T. Nakajima, Y. Honda, O. Kitao, H. Nakai, T. Vreven, J. A. Montgomery Jr., J. E. Peralta, F. Ogliaro, M. Bearpark, J. J. Heyd, E. Brothers, K. N. Kudin, V. N. Staroverov, R. Kobayashi, J. Normand, K. Raghavachari, A. Rendell, J. C. Burant, S. S. Iyengar, J. Tomasi, M. Cossi, N. Rega, J. M. Millam, M. Klene, J. E. Knox, J. B. Cross, V. Bakken, C. Adamo, J. Jaramillo, R. Gomperts, R. E. Stratmann, O. Yazyev, A. J. Austin, R. Cammi, C. Pomelli, J. W. Ochterski, R. L. Martin, K. Morokuma, V. G. Zakrzewski, G. A. Voth, P. Salvador, J. J. Dannenberg, S. Dapprich, A. D. Daniels, Ö. Farkas, J. B. Foresman, J. V. Ortiz, J. Cioslowski and D. J. Fox, Gaussian 09, Revision A.02, Gaussian, Inc., Wallingford, CT, 2009.

24 S. Grimme, J. Comput. Chem., 2006, 27, 1787.

25 R. Krishnan, J. S. Binkley, R. Seeger and J. A. Pople, J. Chem. Phys., 1980, 72, 650.

26 A. D. McLean and G. S. Chandler, J. Chem. Phys., 1980, 72, 5639.

27 J-P. Blaudeau, M. P. McGrath, L. A. Curtiss and L. Radom, J. Chem. Phys., 1997, 107, 5016.

28 B. I. Dunlap, J. Chem. Phys., 1983, 78, 3140.

29 B. I. Dunlap, THEOCHEM, 2000, 529, 37.

30 A. D. Becke, J. Chem. Phys., 1997, 107, 8554.

31 S. Grimme, J. Antony, S. Ehrlich and H. Krieg, J. Chem. Phys., 2010, 132, 154104.

32 Á. Vázquez-Mayagoitia, C. D. Sherrill, E. Aprà and B. G. Sumpter, J. Comput. Chem., 2010, 6, 727.
33 D. Josa, J. R. Otero and E. M. Cabaleiro Lago, Phys. Chem. Chem. Phys., 2011, 13, 21139.

34 P. I. Nagy and P. W. Erhardt, J. Phys. Chem. B, 2012, 116, 5425.

35 A. B. González-Pérez, R. Álvarez, O. N. Faza, Á. R. de Lera and J. M. Aurrecoechea, Organometallics, 2012, 31, 2053.

36 J. P. Perdew, K. Burke and M. Ernzerhof, Phys. Rev. Lett., 1996, 77, 3865.

37 J. P. Perdew, K. Burke and M. Ernzerhof, Phys. Rev. Lett., 1997, 78, 1396.

38 C. Adamo and V. Barone, J. Chem. Phys., 1999, 110, 6158.

39 A. V. Marenich, S. V. Jerome, C. J. Cramer and D. G. Truhlar, J. Chem. Theor. Comput., 2012, 8, 527.

40 A. V. Marenich, C. J. Cramer and D. G. Truhlar, CM5PAC, University of Minnesota, Minneapolis, 2011.

41 F. L. Hirshfeld, Theor. Chim. Acta, 1977, 44, 129.

42 J. P. Ritchie, J. Am. Chem. Soc., 1985, 107, 1829.

43 J. P. Ritchie and S. M. Bachrach, J. Comput. Chem., 1987, 8, 499-509.

44 E. R. Johnson, S. Keinan, P. Mori-Sánchez, J. ContrerasGarcía, A. J. Cohen and W. Yang, J. Am. Chem. Soc., 2010, 132, 6498.

45 J. C. Ma and D. A. Dougherty, Chem. Rev., 1997, 97, 1303.

46 M. O. Sinnokrot and C. D. Sherrill, J. Am. Chem. Soc., 2004, 126, 7690.

47 E. G. Hohenstein and C. D. Sherrill, J. Phys. Chem. A, 2009, 113, 878. 